\section{ce}

The findings pinpoint glucose levels as having a key role in influencing intestinal barrier function

\title{
A breach in the intestinal barrier during hyperglycaemia
}

New research published in Science demonstrates that hyperglycaemia is linked to and drives intestinal barrier dysfunction and the resultant risk of systemic dissemination of enteric infection in animal models. The findings pinpoint glucose levels as having a key role in influencing intestinal barrier function, and highlight a potential mechanism for the altered intestinal epithelial integrity observed in the metabolic syndrome.

"The obesity pandemic is one of the most urgent threats to human health worldwide," notes author Eran Elinav. "While adiposity itself is typically not life-threatening, it is accompanied by numerous disease manifestations in multiple organs across the body, including chronic inflammation and enhanced susceptibility to infection," he adds. With this aspect in mind and because obesity is associated with dysfunction of the intestinal epithelium, Elinav and colleagues wanted to explore the

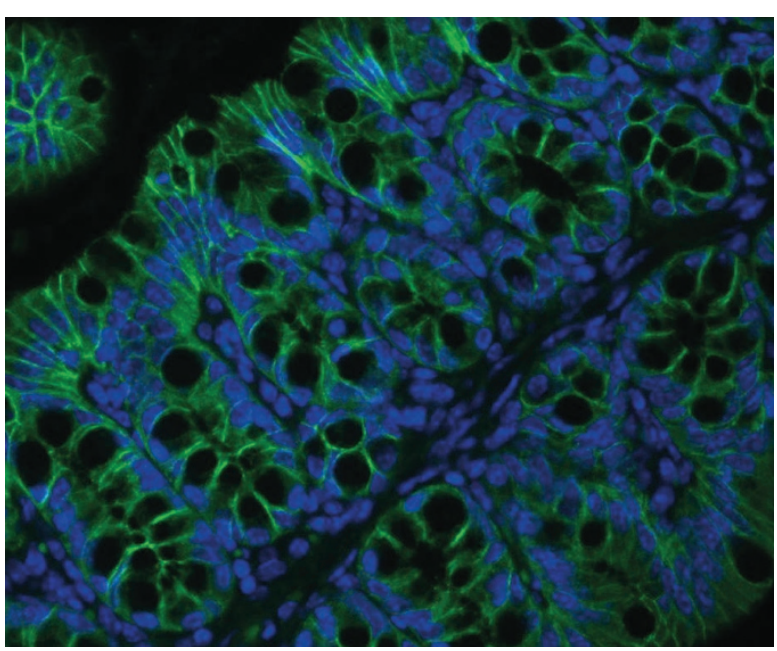

Mouse colonic tissue (E-cadherin, green; DAPI, blue). Courtesy of E. Elinav, Weizmann Institute of Science, Israel. molecular and cellular mechanisms underlying this latter phenomenon.

In a series of experiments using mouse models of obesity or altered leptin signalling, the researchers established that obesity is associated with intestinal barrier dysfunction and increased susceptibility to systemic spread of enteric infection by the mouse pathogen Citrobacter rodentium, but was not a requirement. Interestingly, a common feature of the various mouse models with increased susceptibility to C. rodentium infection was the occurrence of elevated blood glucose levels, which led the authors to explore hyperglycaemia as a possible predisposing factor to gut barrier dysfunction.

Hyperglycaemia in the absence of obesity was induced by administration of streptozotocin (STZ) in mice, and STZ-treated mice developed severe $C$. rodentium infection with systemic bacterial translocation and spread. STZ treatment also led to dysfunction of intestinal epithelial adherens junctions alongside systemic dissemination of microbial products (pattern-recognition receptor (PRR) ligands).

Further analysis of Caco-2 cells (intestinal epithelial cells) in vitro revealed that tight junction integrity (assessed via tight junction protein $\mathrm{ZO}-1$ staining) changed in response to the addition of glucose in the culture medium in a dose-dependent and time-dependent manner, visualized as increased tortuosity (twisting) or altered appearance of cell-cell junctions. Moreover, RNA sequencing analysis of purified intestinal epithelial cells from STZtreated mice and vehicle-treated controls demonstrated global reprogramming of the epithelial transcriptome in hyperglycaemic mice ( $>1,000$ genes differentially expressed), of which the majority were in genes involved in metabolic pathways, particularly $N$-glycan biosynthesis and pentose-glucuronate interconversion.

Notably, this epithelial reprogramming by hyperglycaemia was shown to involve glucose metabolism and especially glucose transporter 2 (GLUT2). In mice lacking GLUT2 specifically from intestinal epithelial cells, STZ did not induce major transcriptional reprogramming in the intestinal epithelium, and integrity of tight and adherens junction complexes was retained. Finally, in 27 healthy individuals, blood glucose levels (as measured by haemoglobin $A_{1 c}$ ) were strongly correlated with serum levels of PRR ligands, but other features of metabolic disease (BMI) were not.

"Our work suggests that there is a direct effect of high glucose levels on the metabolic and transcriptional wiring of epithelial cells, which we are currently investigating in detail," explains Elinav. "This mechanism might be relevant beyond obesity and [might] also play a role in other diseases that have been associated with a defective intestinal barrier, such as nonalcoholic fatty liver disease."

Katrina Ray

ORIGINAL ARTICLE Thaiss, C. A. et al. Hyperglycemia drives intestinal barrier dysfunction and risk for enteric infection. Science https://doi.org/10.1126/science.aar3318 (2018) FURTHER READING Odenwald, M. A. \& Turner, J. R. The intestinal epithelial barrier: a therapeutic target? Nat. Rev. Gastroenterol. Hepatol. 14, 9-21 (2017) 